\title{
Numerical Simulation on Gas-liquid Flow in Mechanical-Gas Injection Coupled Stirred System
}

\author{
Pin SHAO, Ting-an ZHANG, ${ }^{*}$ Zimu ZHANG and Yan LIU \\ School of Materials and Metallurgy, Northeastern University, Shenyang, 110819 People's Republic of China. \\ (Received on January 22, 2014; accepted on April 2, 2014)
}

\begin{abstract}
Based on Euler-Euler approach, a mathematical model is established to describe gas and liquid twophase flow in the mechanical-gas injection coupled stirred system for steelmaking, and the effects of different location and rotation speed of impeller, and gas flow rate on the bubble dispersion, gas total volume and mixing time in the bath were studied. The results show that the predicted bubbles dispersion and mixing time agree well with the experimental photos and measured data. With the increasing of impeller eccentricity, the bubbles dispersion become more better, and the mixing time decreases, while the gas total volume first decreases and then increases. With the increasing of rotation speed of impeller, the bubble dispersion becomes better, and the gas total volume the mixing efficiency also increases. But these effects become weak when the rotation speed exceed $200 \mathrm{rpm}$. With the increasing of gas flow rate, the bubble dispersion remain basically unchanged, and the gas total volume gradually increase, but the mixing time would rapidly increase. It is recommended to use the impeller placed at radial position of $0.4 R$ ( $R$ is the radius of bath), rotation speed of $200 \mathrm{rpm}$, and gas flow rate not exceeding $2.0 \mathrm{Nm}^{3} / \mathrm{h} \mathrm{for}^{\circ}$ present system.
\end{abstract}

KEY WORDS: mechanical-gas injection coupled stirred; gas-liquid flow; bubble dispersion; mixing time; numerical simulation.

\section{Introduction}

In the steelmaking process, the high efficiency of desulfurization become more and more important with the increase of demand for high quality and ultra-low sulfur grade steel, such as heavy plates and linepipe steel. Currently, hot metal pretreatment is the key procedure to get high purity and desulfurization steel, and can be categorized into two methods, namely mechanical stirring and gas injection, respectively. These two methods have their own advantages and disadvantages.

For the mechanical stirring in hot metal desulfurization process, the solid lime is added as flux and emulsified into molten iron using a vortex formed by the stirrer. Many researchers ${ }^{1-5)}$ investigated the desulfurization mechanism of solid lime, and indicated that desulfurization reaction rate using $\mathrm{CaO}$ powder was slow, owing to the fact that the mass transfer in the solid phase is the rate-controlling step and the interfacial layer inhibits the mass transfer of sulfur. ${ }^{5)}$ Furthermore, the amount of slag would be generated during the process of low sulfur steel production with this method, which easily causes iron loss and trouble in the slag skimming process.

For the gas injection in hot metal desulfurization process, Mg-based desulphurization agent is injected into hot metal with a nitrogen or argon carrier gas, and the mechanism and

* Corresponding author: E-mail: zta2000@163.net DOI: http://dx.doi.org/10.2355/isijinternational.54.1507 behavior of desulfurization have been investigated by many researchers. ${ }^{6-13)}$ Compared with calcium-based agents, the magnesium vapor produced by the evaporation of magnesium metal or by aluminothermic reduction of magnesium oxide under high temperature, is more superior for hot metal desulfurization. However, most of the previous research have showed that the desulfurization efficiency of magnesium-based was still very low in desulfurizing the molten iron to an ultralow sulfur level. Ashton et al. ${ }^{9)}$ showed that the desulfurization efficiency approximately reduced from 60 to 13 pct with the average sulfur concentration decreasing from 900 to $200 \mathrm{ppm}$. Hiraga et al. ${ }^{10)}$ indicated that the average desulfurization efficiency was only about 14 pct with the average sulfur concentration decreasing from 250 to $20 \mathrm{ppm}$. The main reasons of low efficiency of desulfurization with this method is that the large bubbles formed by magnesium vapor, disperse only locally in the bath center and rise up very rapidly with the upward mixed flow of gas and liquid. As a result, their residence time in the bath becomes short to make the gas-liquid contact very poor.

In order to improve desulfurization efficiency, the coupled method of gas injection and mechanical stirring have been proposed by Zhang and his coworkers. ${ }^{14-16)}$ In this approach, the bubbles of magnesium vapor could be generated from the pellets of dolomite and reductant in hot metal, and then that would obviously disperse and breakup under the strong turbulence flow created by the rotation of the impeller, which in turn increase magnesium bubble utilization. In previous works, water model experiments was 
adopted to study the bubble behavior in mechanism-gas injection stirring system. But there are still few mathematical model to be developed, and the turbulent flown filed, bubble local distribution and gas total volume stayed in the mechanical-gas injection coupled stirring bath have not been systematically described, which are very important for improving gas-liquid contact area, species mixing and desulfurization efficiency.

The objectives of present work are to present a mathematical model to describe the gas injection and mechanical stirring coupled system for hot metal pretreatment based on Euler-Euler approach, and to predict the gas volume fraction distribution, the flow filed and species mixing process reasonably. The effects of impeller location and rotation speed, and gas flow rate on the present systems are investigated, and a proper operating factors are proposed.

\section{Mathematical Models}

\subsection{Eulerian Multiphase Hydrodynamic Equations}

Based on Euler-Euler approach, the mass and momentum balance equations are used for each phase separately. The interaction forces between the gas and liquid two-phase were considered as momentum exchange source terms in the momentum equations.

Mass conservation:

$$
\frac{\partial}{\partial t}\left(\alpha_{k} \rho_{k} \bar{u}_{k}\right)+\nabla \cdot\left(\alpha_{k} \rho_{k} \bar{u}_{k}\right)=0
$$

Where, $\rho_{k}, \alpha_{k}$ and $\bar{u}_{k}$ are the density, volume fraction and phase averaged velocity of liquid phase $(k=l)$ and the gas phase $(k=g)$, respectively.

Momentum conservation:

$$
\begin{aligned}
& \frac{\partial}{\partial t}\left(\alpha_{k} \rho_{k} \bar{u}_{k}\right)+\nabla \cdot\left(\alpha_{k} \rho_{k} \bar{u}_{k} \bar{u}_{k}\right)= \\
& -\alpha_{k} \nabla p+\nabla \cdot\left(\alpha_{k} \mu_{e f f}\left(\nabla \bar{u}_{k}+\left(\nabla \bar{u}_{k}\right)^{T}\right)\right)+\alpha_{k} \rho_{k} \bar{g}+F_{D} \\
& \mu_{e f f}=\mu_{l}+\mu_{t} \\
& \mu_{t}=C_{\mu} \rho_{l} \frac{k_{l}^{2}}{\varepsilon_{l}}
\end{aligned}
$$

Where, $\bar{g}$ is the acceleration due to gravity, and $\mu_{l}, \mu_{t}, \mu_{\text {eff }}$ are the liquid molecular viscosity, turbulent viscosity, effective viscosity respectively, $p$ is the pressure, which is shared by both the phase, and $F_{D}$ is the interfacial momentum exchange term between gas and liquid due to drag force. Other interphase force are neglected in the present study based on the report of Khopkar et al. ${ }^{17)}$

The drag force between the gas and liquid phases is represented by the following equation

$$
\begin{gathered}
F_{D}=K_{g l}\left(\bar{u}_{g}-\bar{u}_{l}\right) \ldots \ldots \\
K_{g l}=\frac{3 \alpha_{g} \alpha_{l} \rho_{l} C_{D}}{4 d_{g}}\left|\bar{u}_{g}-\bar{u}_{l}\right|
\end{gathered}
$$

where, $K_{g l}$ is the interphase momentum exchange coefficient due to drag force and $d_{g}$ is the diameter of the bubbles. According to the study of Sano and Mori, ${ }^{18)}$ the bubble diameter is calculated as follows:

$$
d_{b}=\left[\left(\frac{6 \sigma d_{0}}{\rho_{l} g}\right)^{2}+0.0248\left(Q_{g}^{2} d_{0}\right)^{0.867}\right]^{\frac{1}{6}}
$$

where, $Q_{g}$ is the gas flow rate, $d_{0}$ is the nozzle diameter, and $\sigma$ is the gas-liquid surface tension coefficient.

$C_{D}$ is the drag force coefficient, and the drag model of Kolve $^{19)}$ was selected in present model, as it takes into account the drag of distorted bubbles for different flow regime. $C_{D v i s}, C_{D d i s}$ and $C_{D c a p}$ represent the drag force coefficient calculated in the viscous regime, distorted bubbles regime and capped bubbles regime, respectively.

$$
\begin{aligned}
& C_{\text {Dvis }}=24 / \operatorname{Re}\left(1+0.1 \operatorname{Re}^{0.75}\right) \\
& C_{\text {Ddis }}=2 / 3\left(\frac{\left(g \rho_{l}\right)^{0.5} d_{g}}{\sigma^{0.5}}\right)\left(\frac{1+17.67\left(1-\alpha_{g}\right)^{1.286}}{18.67\left(1-\alpha_{g}\right)^{1.5}}\right)^{2} \\
& C_{\text {Dcap }}=3 / 8\left(1-\alpha_{g}\right)^{2}
\end{aligned}
$$

In the viscous regime, i.e. $C_{D d i s}<C_{D v i s}$ the drag coefficient $C_{D}$ is computed as

$$
C_{D}=C_{D v i s}
$$

In the distorted bubbles regime, i.e. $C_{D v i s}<C_{D d i s}<C_{D c a p}$, the drag coefficient $C_{D}$ is computed as

$$
C_{D}=C_{\text {Ddis }}
$$

In the capped bubbles regime, i.e. $C_{D \text { dis }}>C_{D v i s}$, the drag coefficient $C_{D}$ is computed as

$$
C_{D}=C_{\text {Dcap }}
$$

\subsection{Turbulence Models}

The $k-\varepsilon$ model is originally used to solve the single-phase turbulence, and for the gas and liquid two-phase flow, turbulence modeling such as bubble-induce turbulence and interaction source terms between two phases turbulence are still the main unresolved problems.

In order to account for the additional turbulent kinetic energy produced by the bubble motion, the bubble-induced turbulence is generally accounted for by source terms appearing in the equations of $k-\varepsilon$.

$$
\begin{gathered}
\frac{\partial}{\partial t}\left(\alpha_{l} \rho_{l} k\right)+\nabla \cdot\left(\alpha_{l} \rho_{l} \vec{u}_{l} k\right)= \\
\nabla \cdot\left(\alpha_{l} \frac{\mu_{t, l}}{\sigma_{k}} \nabla k\right)+\alpha_{l} G_{k, l}+\alpha_{l} G_{b}-\alpha_{l} \rho_{l} \varepsilon \\
\frac{\partial}{\partial t}\left(\alpha_{l} \rho_{l} \varepsilon\right)+\nabla \cdot\left(\alpha_{l} \rho_{l} \vec{u}_{l} \varepsilon\right)= \\
\nabla \cdot\left(\alpha_{l} \frac{\mu_{t, l}}{\sigma_{\varepsilon}} \nabla \varepsilon\right)+\alpha_{l} \frac{\varepsilon}{k}\left(C_{1 \varepsilon}\left(G_{k}+G_{b}\right)-C_{2 \varepsilon} \rho_{l} \varepsilon\right)
\end{gathered}
$$

where, $k$ and $\varepsilon$ are the turbulent kinetic energy and the dissipation rate of the liquid phase respectively, $G_{k, l}$ denotes the production of turbulence kinetic energy due to the liquid phase shear-induced, and $G_{b}$ is the additional bubbleinduced turbulent kinetic energy. 


$$
\begin{aligned}
& G_{k, l}=\mu_{t}\left(\nabla \vec{u}_{l}+\left(\nabla \vec{u}_{l}\right)^{T}\right): \nabla \vec{u}_{l} \\
& G_{b}=C_{b} \frac{\mu_{t}}{\mu_{e f f}}\left(\rho_{l}-\rho_{g}\right) g \alpha_{g} \vec{u}_{r e l}
\end{aligned}
$$

where, $C_{b}$ denotes the fraction of bubble-induced energy converted into the liquid phase turbulence. In present work, $C_{\mu}, C_{1 \varepsilon}, C_{2 \varepsilon}, \sigma_{k}, \sigma_{\varepsilon}$ are constants and their values are $C_{\mu}=$ 0.07, $C_{1 \varepsilon}=1.44, C_{2 \varepsilon}=1.92, \sigma_{k}=1.0, \sigma_{\varepsilon}=1.3$.

\subsection{Tracer Transport Equation}

The mixing process in chemical and metallurgical processing vessels could be described by mixing time which is defined as that time when all the local concentrations of tracer reached within 5 pct deviation of the homogeneous value. In order to determine the mixing time in the vessel, the tracer dispersion equation need to be solved and can be expressed as follows

$$
\frac{\partial}{\partial t}(\rho C)+\nabla \cdot\left(\rho \vec{u}_{l} C\right)=\nabla \cdot\left(\frac{\mu_{t}}{S c_{t}}(\nabla C)\right)
$$

where $C$ is the local mass fraction of tracer, $S c_{t}$ is the turbulent Schmidt number and the default $S c_{t}$ is 0.7 .

\subsection{Numerical Scheme and Experimental Work}

In this work, the commercial computational fluid dynamics software fluent 12.0 combined with User-Defined Function (UDF) was used to simulate gas and liquid two-phase flow in the mechanical-gas injection coupled stirred system. The dimensions of water model and other parameters employed in present model are shown in Table 1. Figure 1 shows the boundary conditions and mesh of water model. In present model, a total about 112000 meshes are created to develop the flow computational domain, where about 1100 tetrahedral meshes are adopted around four gas blowing at the bottom tuyeres of impeller, and the structured hexahedral meshes are adopted in the remaining regions. The bottom and side walls were set as no-slip solid walls, and the standard wall function was used to model the turbulence characteristic in the near-wall region. The velocity-inlet was used for four gas blowing, and a free liquid surface was assumed at the top surface, where was outlet for the gas and a free slip wall for the liquid. The bubble diameter was treated as constants and calculated with the Eq. (11). In present work, the sloped swept-back blade impellers named SSB-D impeller was adopted. ${ }^{15)}$ The side wall between the blades was sloped and in addition swept back oppositely to the direction of rotation. The slope angle of the blades was $30 \mathrm{deg}$. The central part of the impeller was in the form of square plate and four blades were attached to the side of the plate, and then a circular disk was put on the top side of the blade to prevent air bubbles rising rapidly.

We used the multiple frame of reference (MFR) approach, which is one of the widely used methods in the literature for simulating the flow field in stirred reactors. The second order equivalent to high-resolution discretization scheme was applied for obtaining the algebraic equations for momentum, volume fraction of individual phases, turbulent kinetic energy, turbulence dissipation rate, etc. To avoid numerical difficulties, the transient calculations were made for two-phase flow starting with converged steady-state single primary phase flow field. The converged solution was assumed when the scaled residuals of all variables were smaller than $10^{-3}$.

In order to validate the model predictions, the corresponding experimental work was done to investigate the bubble behavior and mixing phenomenon in water model experimental system. The bubble behavior and distribution in bath was recorded by a high-speed video camera (500 frame/s), and the mixing process was recorded by electrical conductivity meter to monitor the changes of local conductivity of water with time after the addition of a pulse tracer $(\mathrm{NaCl})$.

\section{Results and Discussion}

It is well known that the liquid flow and bubble dispersion interact each other significantly, and affect the gas-liquid contact area and specie mixing phenomena seriously, which in turn affect the desulfurization efficiency in mechanicalgas injection coupled stirred system. Thus the accurate prediction of hydrodynamics is the basis for describing these behaviors. In present work, the predicted bubble dispersion and mixing time under the action of impeller rotation are

Table 1. The Dimensions of Water Model and Other Parameters Employed in Model.

\begin{tabular}{cc}
\hline Bath diameter & $435 \mathrm{~mm}$ \\
Bath depth & $350 \mathrm{~mm}$ \\
Diameter of holes & $1 \mathrm{~mm}$ \\
Impeller immersion depth & $283 \mathrm{~mm}$ \\
Disk diameter & $220 \mathrm{~mm}$ \\
Rotation speed of impeller & 50 to $150 \mathrm{rpm}$ \\
Gas flow rates & 0.5 and $3.0 \mathrm{~m}^{3} / \mathrm{h}$ \\
Density of water & $1000 \mathrm{~kg} / \mathrm{m}^{3}$ \\
Density of gas & $1.225 \mathrm{~kg} / \mathrm{m}^{3}$ \\
Molecular viscosity of water & $0.001 \mathrm{~Pa} \mathrm{~s}$ \\
Surface tension between water and gas & $0.073 \mathrm{~N} / \mathrm{m}$ \\
\hline
\end{tabular}

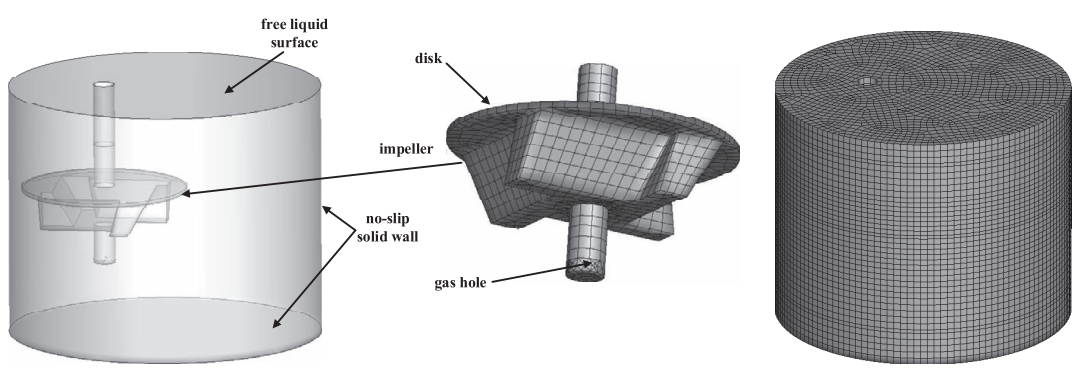

Fig. 1. Boundary conditions of water model and the mesh of impeller. 
compared with experimental photos and measured data, and the effect of impeller location and rotation speed, and gas flow rate on the present systems are investigated as following.

\subsection{Effect of Impeller Eccentricity}

\subsubsection{Bubble Dispersion and Liquid Flow}

Figure 2 shows the effect of different eccentric position of impeller on the bubble dispersion, and the comparison of predicted results by present model with experimental photos. The gas flow rate is $2.0 \mathrm{~m}^{3} / \mathrm{h}$ and the rotation speed is $150 \mathrm{rpm}$. The nozzle immersion depth is $0.283 \mathrm{~m}$, and the eccentricity of the impeller shaft is varied between 0 and 0.4. From this figure, it can be found that when the impeller is located in the center, the bubble is mainly concentrated in the end of the impeller blade and at the bottom of circular disk which was put on the top side of the blade to prevent bubbles rising directly. During the bubble rising process, the bubbles would gradually gathered to the center due to liquid flow. As the impeller is moved away from the center toward the side wall, the bubbles become more and more uniform in the whole bath, and the predicted bubbles dispersion agree well with the measured experimental photos.

In Fig. 3, the effect of different eccentric position of impeller on the liquid flow is illustrated. From this figure, it is observed that when the impeller is located in the center, the liquid flow filed is symmetric relative to impeller in the whole vessel, and the high velocity zone appears in the vicinity of the wall, and the low velocity zone appears in the central of the pool. Furthermore, it is noted that there is a strong interaction between liquid flow filed and the distri-

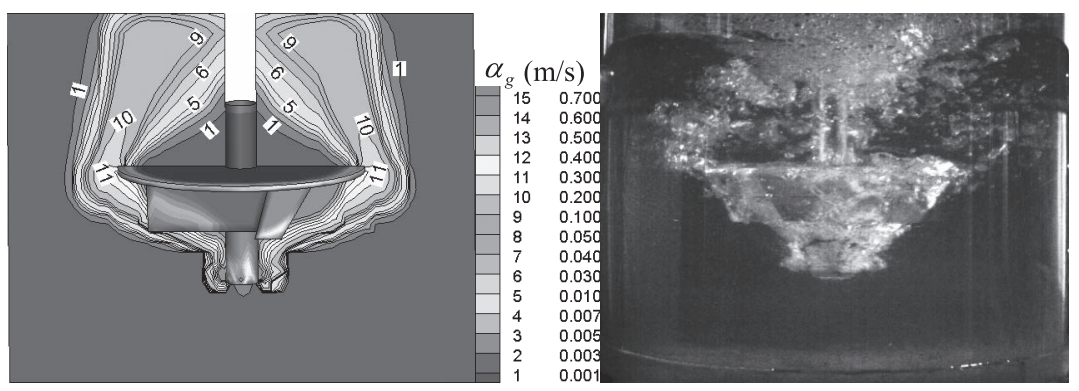

(a)
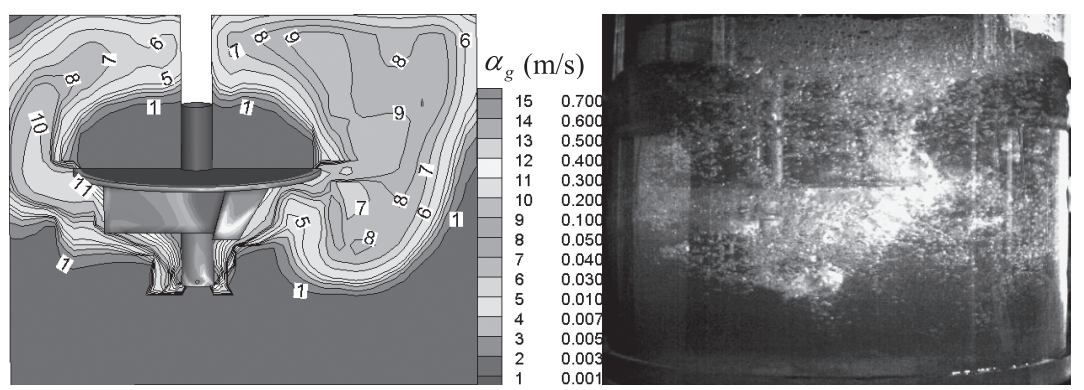

(b)
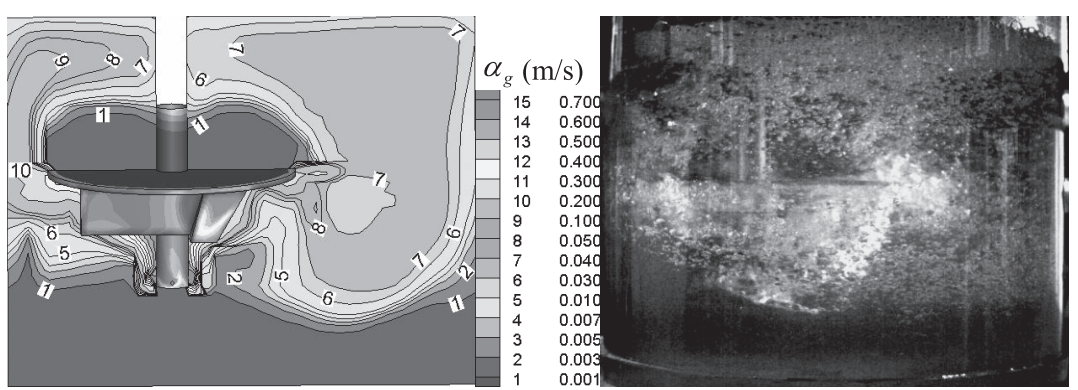

(c)
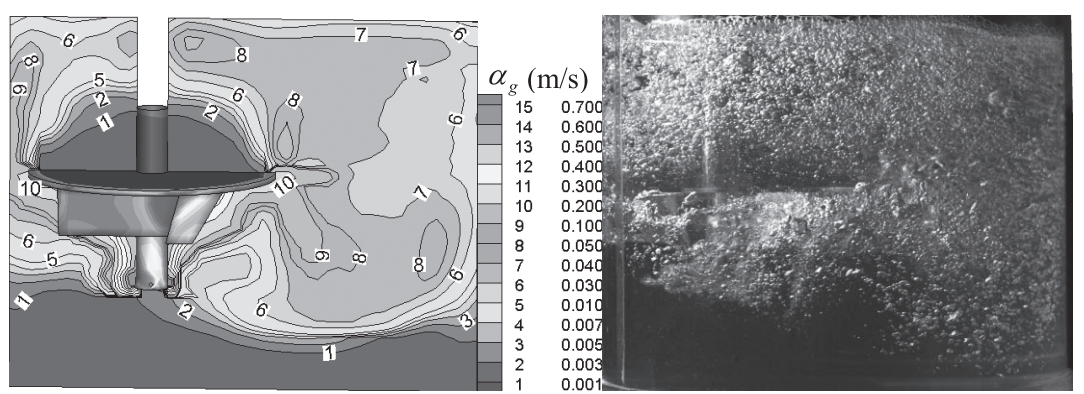

(d)

Fig. 2. Comparison of the predicted local gas volume fraction with experimental photos in the bath with different eccentric position of impeller. The eccentricity is (a) 0 , (b) 0.2 , (c) 0.3 , (d) 0.4 , respectively. 


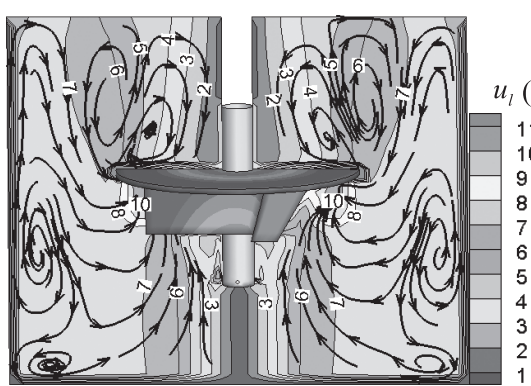

(a)

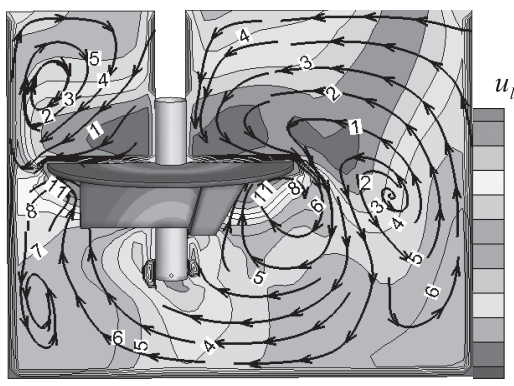

(c)

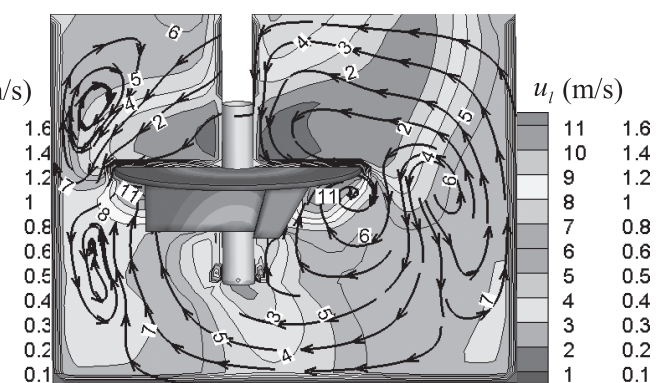

(b)

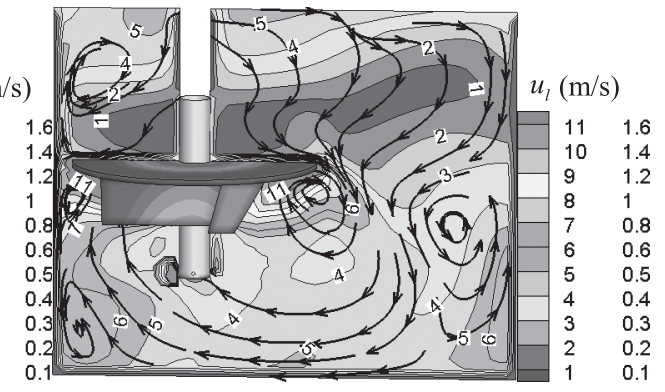

(d)

Fig. 3. Predicted liquid flow filed in the bath with different eccentric position of impeller. The eccentricity is (a) 0 , (b) 0.2 , (c) 0.3 , (d) 0.4 , respectively.

bution of gas volume fraction in vessel. On the one hand, the upwelling liquid flow driven by bubble buoyance are formed in bubbly plume zone, and accordingly the circulations are established around the bubble plume. On the other hand, during the bubble rising process, the bubbly plume would gradually bend to the center due to extrusion of the outer circulation produced by impeller rotation, as shown in Fig. 1(a). When the impeller is moved away from the center, the symmetric flow is destroyed, and several large circulation were formed and coupled to each other, which would facilitate components transport in the whole bath. Furthermore, the downward flow is gradually formed in the upper zone of vessel with the increasing of impeller eccentricity, which would facilitate to prevent air bubbles floating quickly.

\subsubsection{Gas Total Volume}

It is well known that under the same condition of gas blowing, with the increasing of bubbles residence time in bath, the gas total volume in bath would become larger, which in turn facilitate gas-liquid reaction efficiency. The gas total volume $\theta$ can be calculated by integrating the local gas volume fraction $\alpha_{g}$ and cell volume $V_{\text {cell }}$ throughout the bath:

$$
\theta=\int \alpha_{g} d V_{\text {cell }}
$$

Figure 4 shows the predicted gas total volume $\theta$ in the bath under different eccentricity of impeller varying from 0 to 0.4 . It can be seen from this figure that the gas volume in the bath first decreases, and then increases with the movement of the impeller along the radial direction from center to side wall. When the eccentricity of 0.4 is adopted, the bubble was blocked by the downward flow produced by impeller, as shown in Fig. 3(d), and the total gas volume $\theta$ is the maximum. When the eccentricity of 0.2 is adopted, that is the minimum.

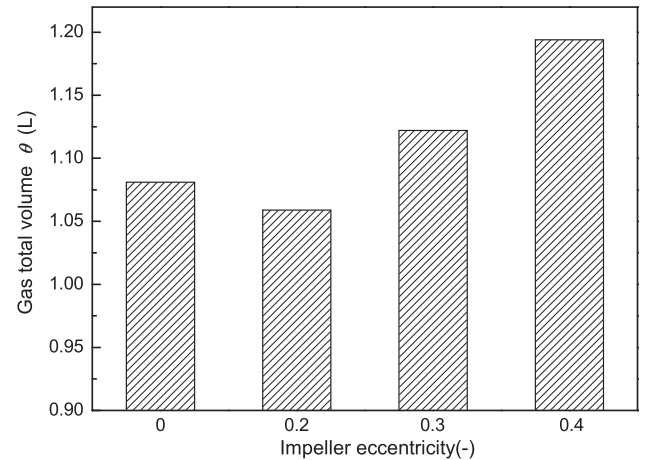

Fig. 4. Predicted gas total volume in the bath with different eccentric position of impeller.

\subsubsection{Mixing Phenomenon}

The mixing degree determining the efficiency of metallurgical reactions in bath can be evaluated from a degree 95 pct bulk mixing time, which is defined as the maximum time when all the local concentrations of tracer reach within 5 pct deviation of the homogeneous value. Several investigations ${ }^{20,21)}$ have concluded that mixing time is affected by the tracer addition location and monitoring point which should be placed exactly in the dead zone. From Fig. 4, it is observed that the lowest flow velocity zone is always at the top of circular disk. In present work, as shown in Fig. 5, multiple tracer addition locations (A1 to A5) and tracer monitoring points (M1 to M6) are adopted to eliminate deviation of mixing time as far as possible.

Figure 6 shows the predicted typical variation of tracer mass concentration of the six monitoring point (M1 to M6) with time after the trace added to point $\mathrm{A} 1$ and $\mathrm{A} 2$ respectively. In this figure, the $C / C_{\text {ave }}$ represents the ratio of the local tracer concentration and the homogeneous value. It is observed that the times that the concentration of each monitoring point reaches within 5 pct deviation of the homoge- 
neous value, are different. For example, when the tracer is added to point $\mathrm{A} 1$, the monitoring point $\mathrm{M} 2$ is the last one reaching within 5 pct deviation of the homogeneous value, and the mixing time tA 1 is $8.5 \mathrm{~s}$. When the trace is added to point A5, the monitoring point M1 is the last one, and the mixing time tA2 is $6.13 \mathrm{~s}$. but overall, in the present mechanical-gas injection coupled stirred system, the mixing process is rapidly, and the effect of locations of tracer addition and monition on the mixing time is relatively weak.

Figure 7 shows the measured and predicted typical variation of tracer dimensionless concentration with time in bath. In the experiments, the multiple locations is hardly monitored by conductivity probe simultaneously due to experimental limitations, thus only the tracer monitoring points M5 and tracer addition points A2, as shown in Fig. 5 , are adopted to measure the mixing time. A pulse tracer

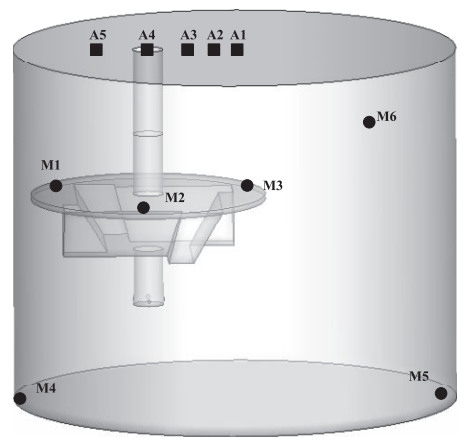

Fig. 5. Arrangement of tracer addition points (A1 to A5) and tracer monitoring points (M1 to M6) in bath.

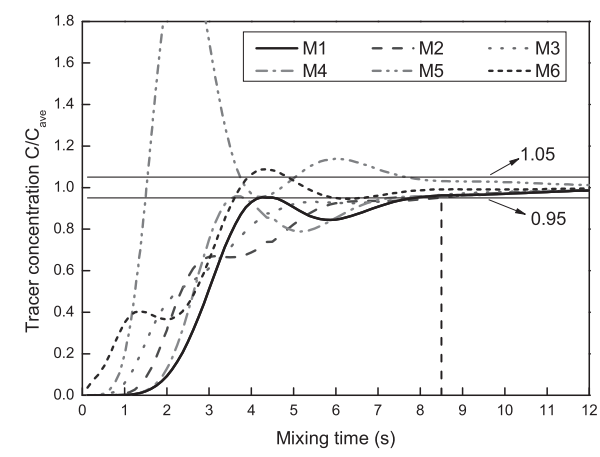

(a)
(30 $\mathrm{mL}$ saturated solution of $\mathrm{NaCl}$ ) was added into the bath in three minutes after the start of gas-mechanical stirring, and then the mixing process was recorded by electrical conductivity meter to monitor the changes of local conductivity of water with time. In Fig. 7 , the $C / C_{a v e}$ represents the ratio between the local tracer conductivity or mass concentration and the homogeneous value, and it can be found from this figure that the predicted result agrees well with the measured data at the same tracer addition and monitoring points. For each case, more than 3 times measurements are performed, and the mean value is taken as the measured mixing time.

Figure 8 shows the effect of different eccentricity of impeller on the mixing times in the bath. In present work, the time $t_{\text {ave }}$, which is the average of the five mixing time $\left(t_{A 1}\right.$ to $\left.t_{A 5}\right)$ predicted by adding the trace on five different point in ladle (A1 to A5), is proposed to represent the mixing efficiency of the bath. $t_{\text {measured }}$ is measured average mixing time in the experiment. From this figure, it can be found that the predicted mixing time agree well with the measured data, and when the impeller is at the center, the mixing time is the maximum, since it is difficult for tracer transport between the two separate symmetric flows in the bath, as shown in Fig. 3(a). When the eccentric impeller is adopted, the average mixing time significantly become short due to well dispersion of bubble and big circulation formed in the whole bath, as shown in Figs. 3(b), 3(c) and 3(d).

Overall, the mixing time with eccentric impeller is significantly shorter than that with central impeller, and with the increasing of eccentricity of impeller, the bubbles become

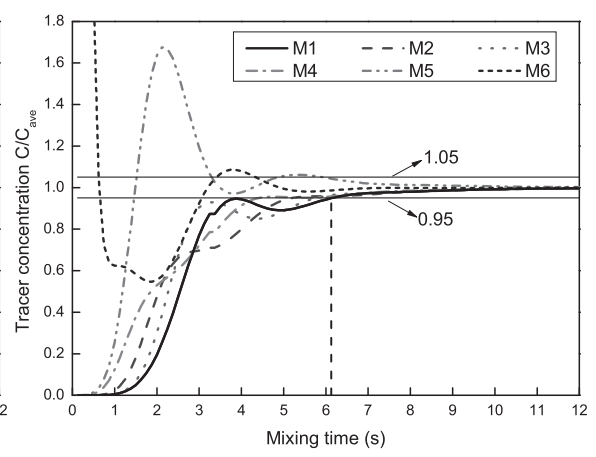

(b)

Fig. 6. Predicted variation of tracer mass concentration of the six monitoring points (M1-M6) with time after the tracer added to (a) point A1 and (b) point A5 respectively.

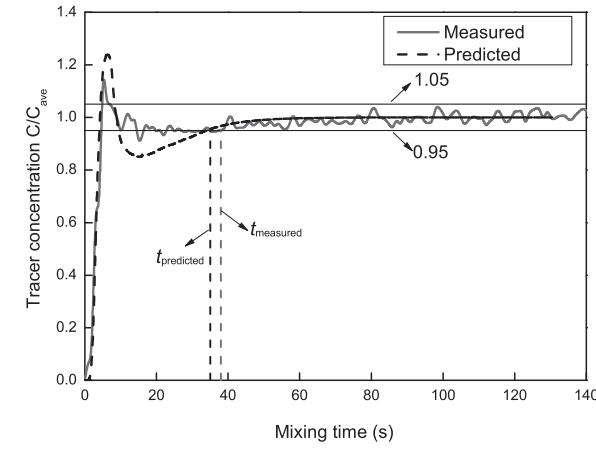

(a)

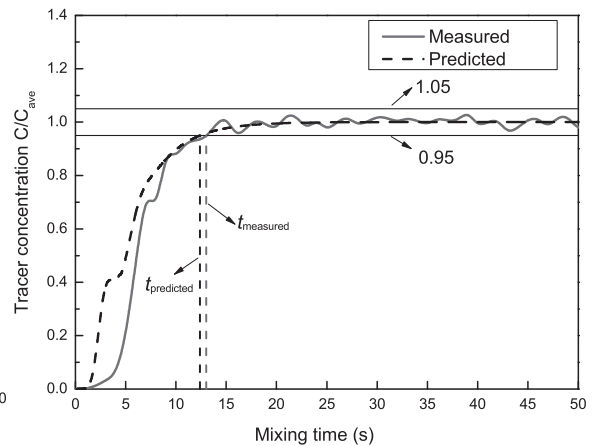

(b)

Fig. 7. Measured variation of tracer conductivity of the monitoring points M5 with time after the tracer added to point A2. The eccentricity of impeller are (a) 0 , and (b) 0.4 , respectively. 
more and more uniform, but the gas total volume first decreases, and then increases. The impeller eccentricity of 0.4 is recommended for present system.

\subsection{Effect of Impeller Rotation Speed}

The effect of rotation speed of impeller on the disrtibution of gas volume fraction and liquid flow filed are given in Figs. 9 and 10, respectively. In these figures, the impeller eccentricity is 0.4 , and the gas flow rate is $2.0 \mathrm{Nm}^{3} / \mathrm{h}$. It can be found from these figures that the predicted bubble distribution in bath argee well with the measured experimental photos under different rotation speed of impeller. When the rotation speed of $50 \mathrm{rpm}$ was adopted, the liquid flow in the whole is relatively slow and has little effect on the bubble

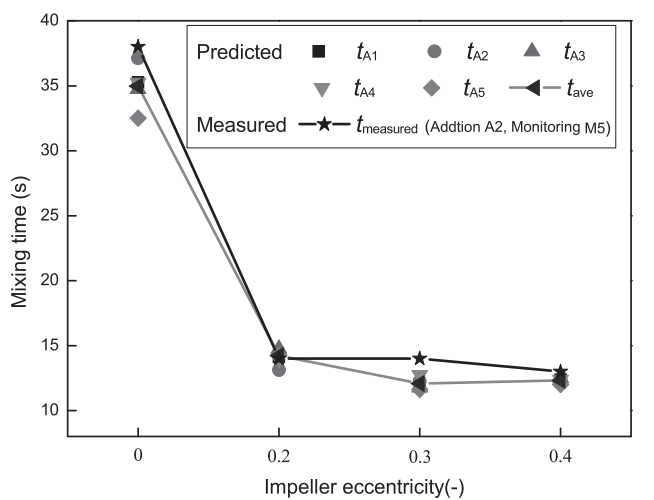

Fig. 8. Predicted and measured mixing time in the bath with different eccentricity of impeller.
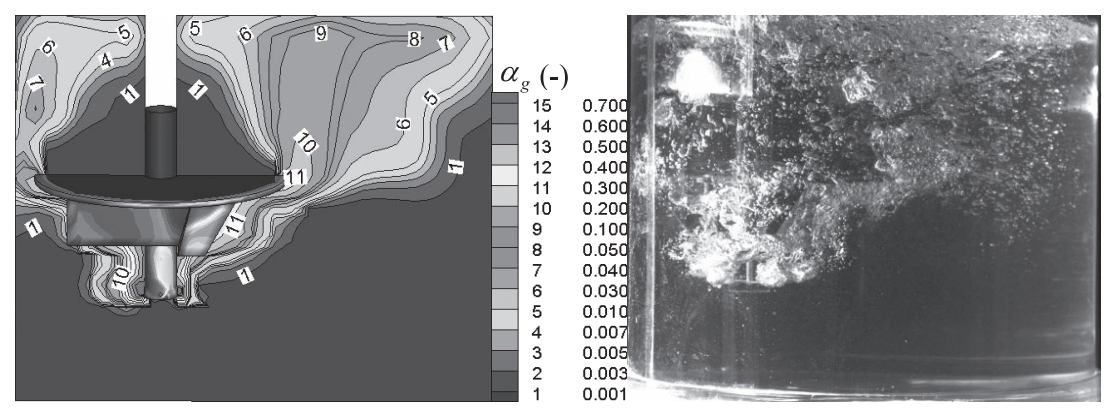

(a)
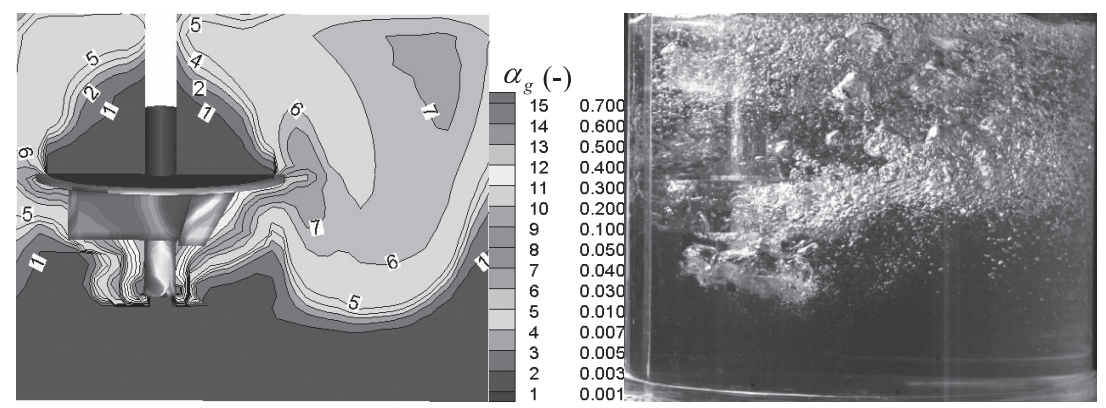

(b)
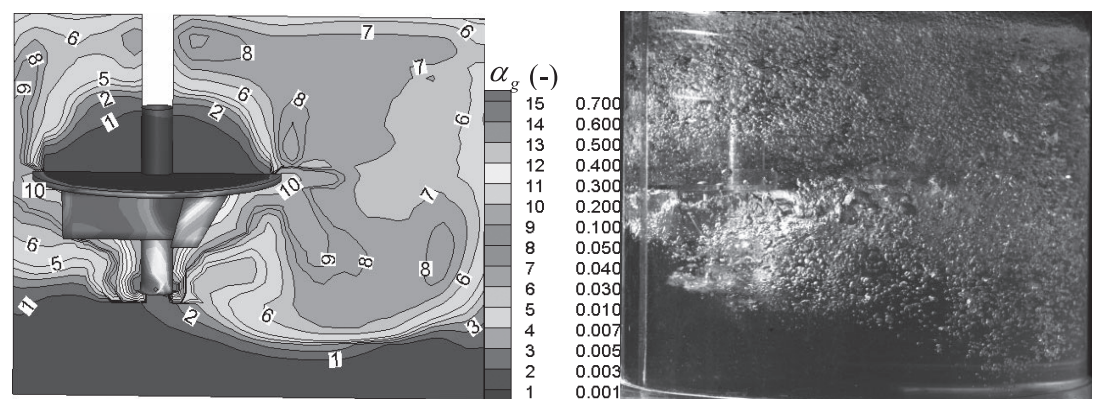

(c)
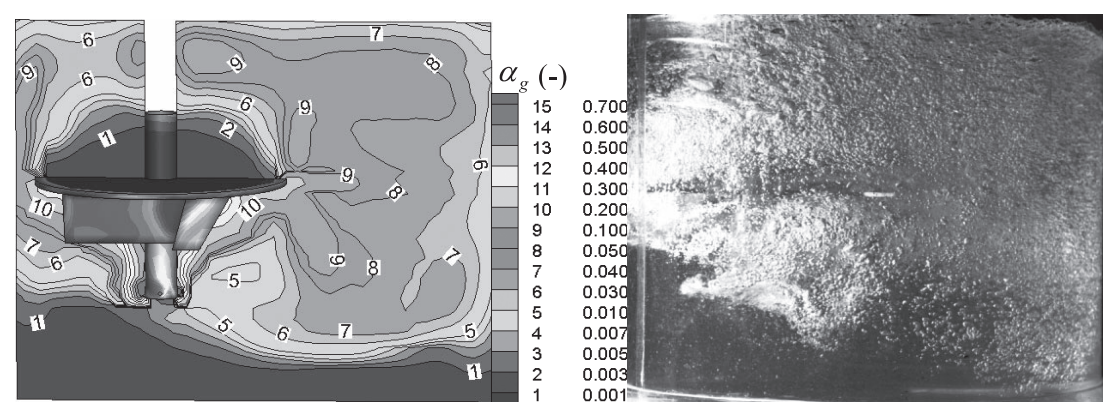

(d)

Fig. 9. Comparison of the predicted local gas volume fraction with experimental photos in the bath with different rotation speed of impeller. The rotation speed is (a) $50 \mathrm{rpm}$, (b) $100 \mathrm{rpm}$. (c) $150 \mathrm{rpm}$, (d) $200 \mathrm{rpm}$, respectively. 


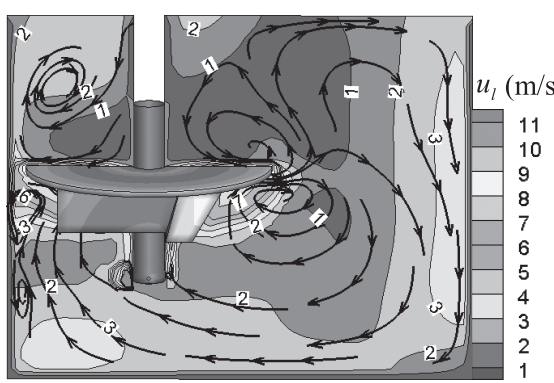

(a)

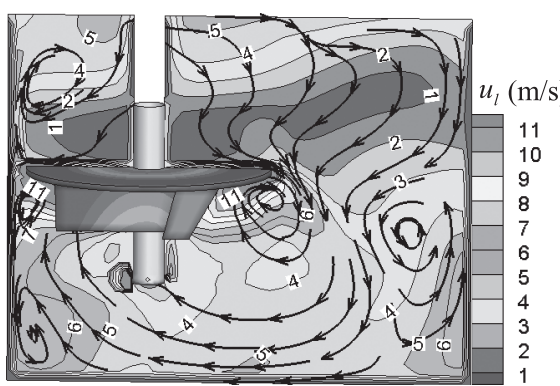

(c)

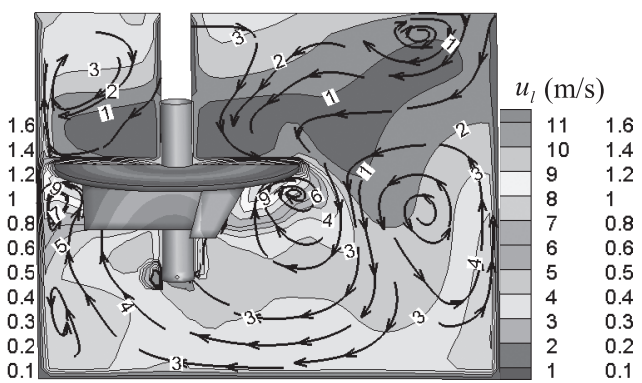

(b)

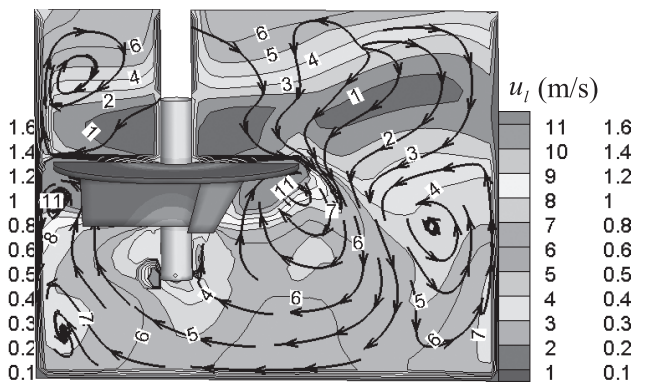

(d)

Fig. 10. Predicted liquid flow in the bath with different rotation speed of impeller. The rotation speed is (a) $50 \mathrm{rpm}$, (b) $100 \mathrm{rpm}$. (c) $150 \mathrm{rpm}$, (d) $200 \mathrm{rpm}$, respectively.

dispersion. The bubble is concentrated in the upper portion of the bath and rarely distributed in the bottom of the bath due to their buoyancy. With the increasing of rotation speed of impeller, the bubble dispersion becomes better in the whole bath. This is mainly because that the liquid flow become more and more intense, and in particular, the downward trend of liquid flow in the upper portion of bath is enhanced, which in turn effectively prevent bubbles floating, as shown in Figs. 10(c) and 10(d). But when the rotiation speed exceeds $150 \mathrm{rpm}$, the bubble distribution in the whole bath changed little.

Figures 11 and $\mathbf{1 2}$ show the effect of rotation speed of impeller on the total gas volume and mixing time in the bath. It is clear from these figures that with the increasing of rotation speed of impeller, the gas total volume incrase due to well bubble dispersion in the whole bath, and the mixing time decreases due to high liquid flow velocity. But when the rotation speed of impeller exceed $200 \mathrm{rpm}$, the total gas volume and mixing time change weakly, and the predicted mixing time agrees well with the measured data.

\subsection{Effect of Gas Flow Rate}

Figures 13 and 14 illustrate the effect of gas flow rate on the local bubble dispersion and liquid flow in the bath. In these figures, the impeller eccentricity is 0.4 , and the rotation speed of impeller $150 \mathrm{rpm}$. It can be found from this figure that with the increasing of gas flow rate, the local value of gas volume fraction in the whole bath increases gradually, but the distribution zone of bubbles remain basically unchanged due to the effect of liquid flow produced by the impeller rotation. the predicted bubble distribution in bath argee well with the measured experimental photos under different gas flow rate. Furthermore, in Fig. 14, it can be also noted that with the increasing of gas flow rate, the liquid flow became weaker, since the interference effect of bubble on the liquid flow filed become intense, and the collisions between bubble and liquid consume liquid flow momentum

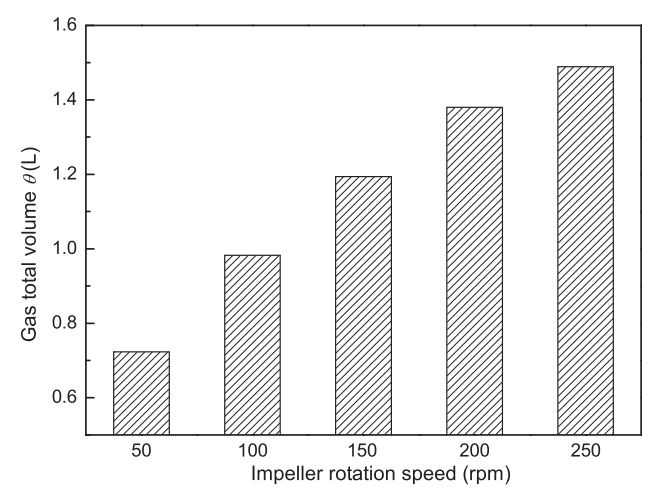

Fig. 11. Predicted gas total volume in the bath with different rotation speed of impeller.

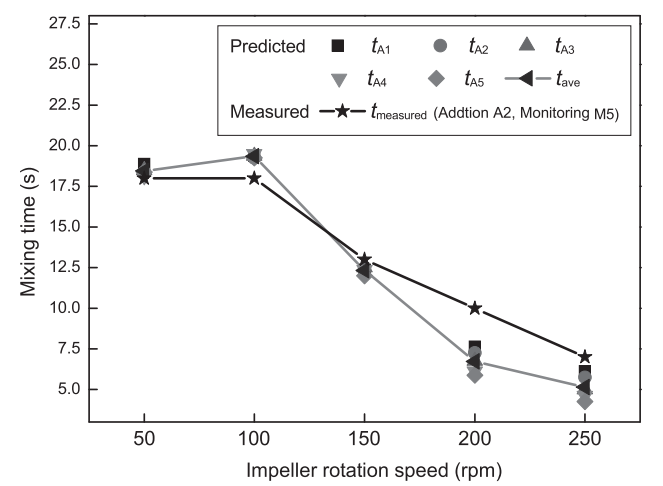

Fig. 12. Predicted mixing time in the bath with different rotation speed of impeller.

produced by mechanically stirring.

Figures 15 and $\mathbf{1 6}$ give the effect of gas flow rate on the gas total volume and mixing time in the bath. From Fig. 15, it can be found that with the increasing of gas flow rate, the gas total volume increase, and when the gas flow rate exceeds $2.0 \mathrm{Nm}^{3} / \mathrm{h}$, the change of gas total volume relatively 


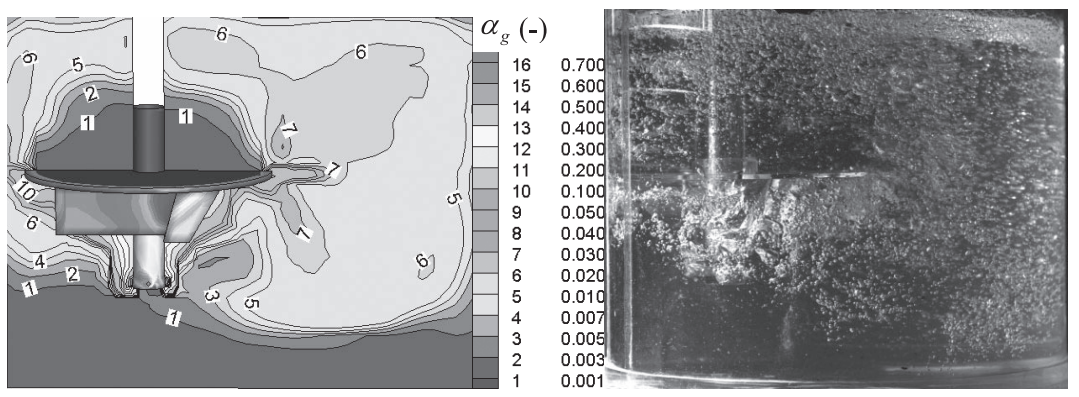

(a)
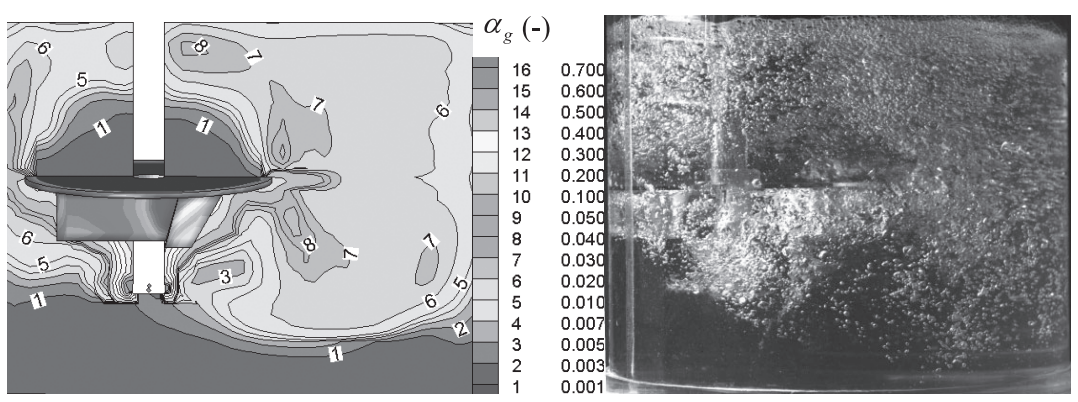

(b)
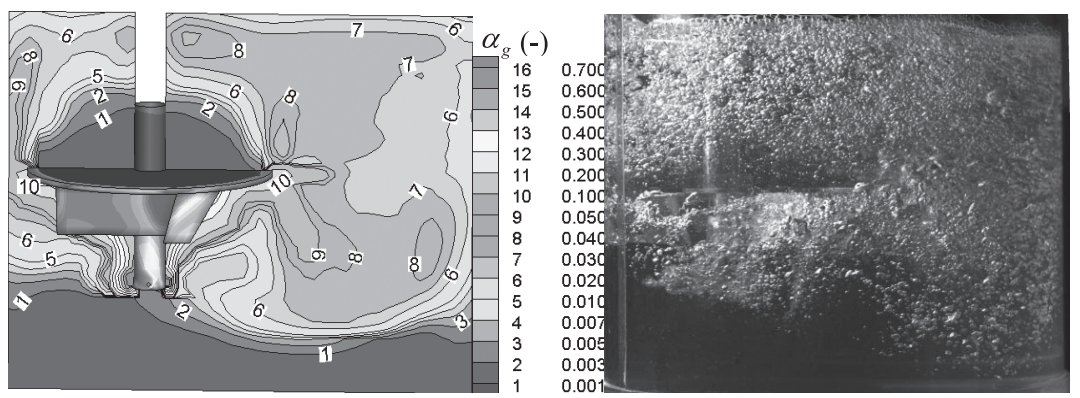

(c)
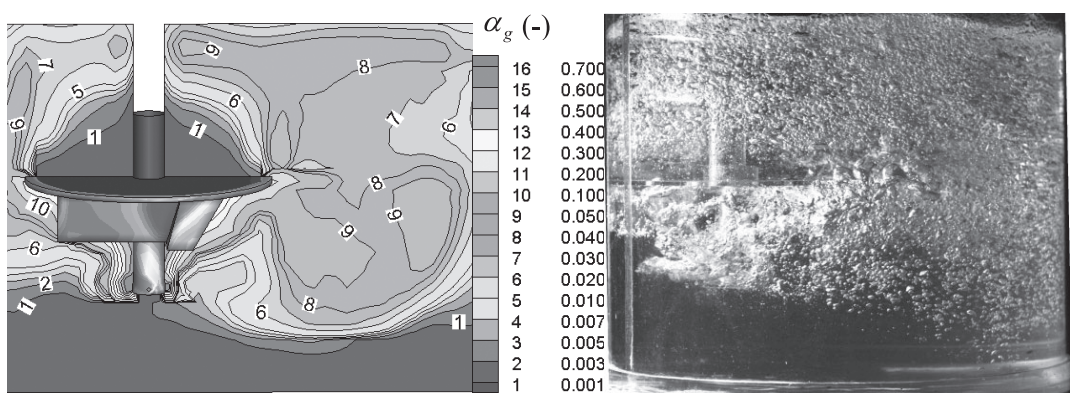

(d)

Fig. 13. Comparison of the predicted local gas volume fraction with experimental photos in the bath with different gas flow rate. The gas flow rate are (a) $0.5 \mathrm{Nm}^{3} / \mathrm{h}$, (b) $1.0 \mathrm{Nm}^{3} / \mathrm{h}$, (c) $1.5 \mathrm{Nm}^{3} / \mathrm{h}$, (d) $2.0 \mathrm{Nm}^{3} / \mathrm{h}$, respectively.

become small. In Fig. 16, it is clear that with the increasing of gas flow rate, the mixing time would rapidly increase, which indicated that excessive gas flow rate would be detrimental to specie mixing efficiency process and chemical reaction rate. The gas flow rate not exceeding $2.0 \mathrm{Nm}^{3} / \mathrm{h}$ is recommended for present system, and the predicted results agree well with the measured data.

\section{Conclusion}

Based on Euler-Euler approach, the gas and liquid twophase flow model was established to describe the mechanical-gas injection coupled stirred system for steelmaking, and the predicted bubbles dispersion and mixing time agree well with the measured experimental photos and measured data. The effects of different location and rotation speed of impeller, and gas flow rate on the bubble dispersion, gas total volume and mixing time in the bath were studied. An optimization had been proposed from the viewpoints of both of bubble dispersion and mixing efficiency.

(1) As the impeller is moved away from the center toward the side wall, the bubbles become more and more uniform, and the gas total volume in the bath first decreases, and then increases. Furthermore, the average mixing time with eccentric impeller is significantly shorter than that with central impeller. Overall, the impeller eccentricity of 0.4 is recommended.

(2) With the increasing of rotation speed of impeller, the 


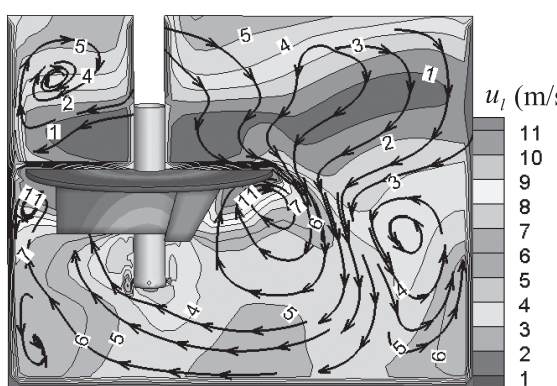

(a)

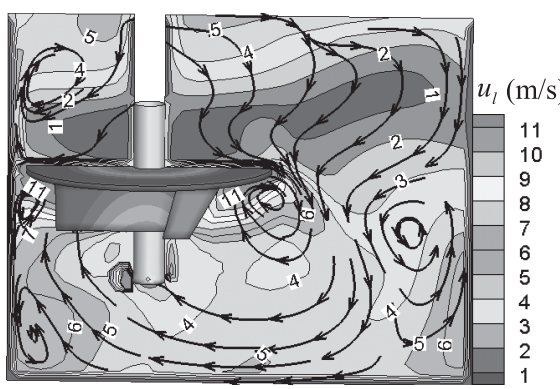

(c)

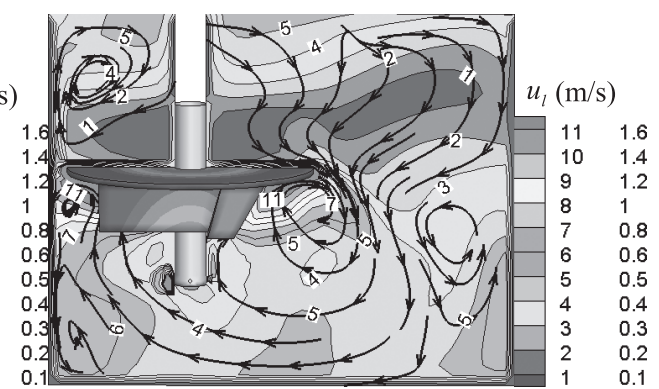

(b)

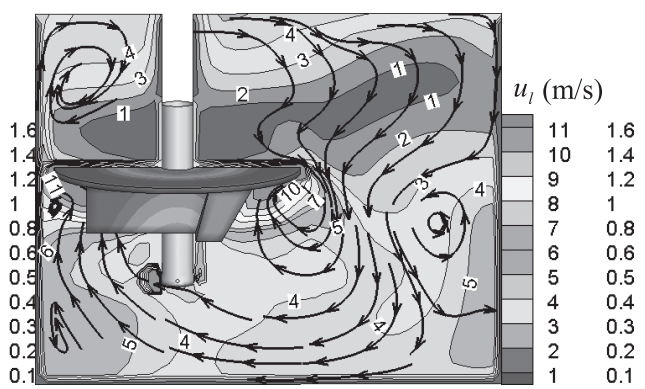

(d)

Fig. 14. Predicted liquid flow in the bath with different gas flow rate. The gas flow rate are (a) $0.5 \mathrm{Nm}^{3} / \mathrm{h}$, (b) $1.0 \mathrm{Nm}^{3} / \mathrm{h}$, (c) $1.5 \mathrm{Nm}^{3} / \mathrm{h}$, (d) $2.0 \mathrm{Nm}^{3} / \mathrm{h}$, respectively.

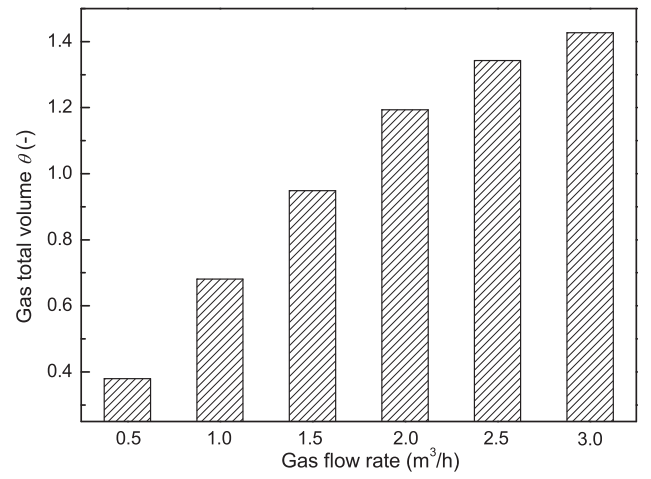

Fig. 15. Predicted gas total volume in the bath with different gas flow rate.

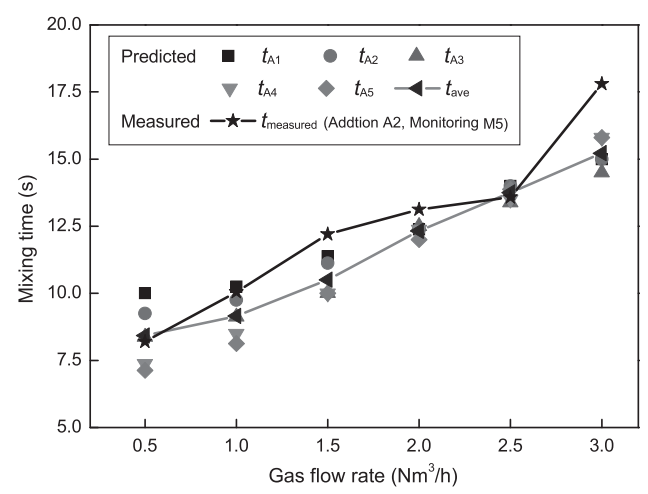

Fig. 16. Predicted Mixing time in the bath with different gas flow rate.

bubble dispersion becomes better, and the gas total volume become larger in the whole bath, in addition, the mixing time also decrease. But these effects become weak when the raotaion speed exceed $200 \mathrm{rpm}$.

(3) With the increasing of gas flow rate, the bubble dispersion remain basically unchanged, and the gas total volume increase, but that change weakly when the gas flow rate exceed 2.0. In addition, the mixing time would rapidly increase with the increasing of gas flow rate, which indicated that excessive gas flow rate would be detrimental to specie mixing efficiency process and chemical reaction rate.

\section{Acknowledgments}

This research was financially supported by the Fundamental Research Funds for the Central Universities (N100302005, N130702001).

\section{REFERENCES}

1) Y. Kawai, K. Mori and Y. San-nomiya: Tetsu-to-Hagané, 61 (1975), 29.

2) M. Ueda, Y. Fujita, K. Nakai, T. Toshimoro and Z. Morita: Tetsu-toHagané, 75 (1989), 58.

3) T. Tanaka, Y. Ogiso, M. Ueda and J. Lee: ISIJ Int., 50 (2010), 1071.

4) Y. Nakai, I. Sumi, H. Matsuno, N. Kikuchi and Y. Kishimoto: ISIJ Int., 50 (2010), 403.

5) K. Takahashi, K. Utagawa, H. Shibata, S. Kitamura, N. Kikuchi and Y. Kishimoto: ISIJ Int., 52 (2012), 10.

6) M. C. Ashton, R. K. Buhr, J. G. Magny and K. G. Davis: Ironmaking Steelmaking, 2 (1975), 111.

7) P. J. Koros, R. G. Petrushka and R. G. Kerlin: Iron Steelmaker, 4 (1977), 34.

8) G. A. Irons and R. I. L. Guthrie: Metall. Mater. Trans. B, 12B (1981), 755.

9) A. Aoyagi, Z. Mukuda, S. Takada and S. Oomiya: CAMP-ISIJ, 7 (1994), 221.

10) Y. Hiraga, K. Gennai and J. Harama: CAMP-ISIJ, 9 (1996), 225.

11) K. Nakanishi, A. Ejima, T. Suzuki and F. Sudo: Tetsu-to-Hagané, 64 (1978), 1323.

12) G. A. Irons and R. I. L. Guthrie: Ironmaking Steelmaking, 8 (1981), 114.

13) J. Yang, K. Okumura, M. Kuwabara and M. Sano: Metall. Mater. Trans. B, 34B (2003), 619.

14) Y. Liu, M. Sano, T. A. Zhang, Q. Wang and J. C. He: ISIJ Int., 49 (2009), 17.

15) Y. Liu, T. A. Zhang, M. Sano, Q.Wang, X. D. Ren and J. C. He: Trans. Nonferrous Met. Soc. China, 21 (2011), 1896.

16) Y. Liu, M. Sano, Q. Wang, T. A. Zhang and J. C. He: CAMP-ISIJ, 20 (2007), 786.

17) A. R. Khopkar, A. R. Rammohan, V. V. Ranade and M. P. Dudukovic: Chem. Eng. Sci., 60 (2005), 2215.

18) M. Sano and K. Mori: Trans. Iron Steel Inst. Jpn., 17 (1976), 344.

19) N. I. Kolev: Multiphase Flow Dynamics 2, Thermal and Mechanical Interactions, 2nd. ed., Springer, Berlin, Germany, (2005).

20) S. Joo and R. I. L. Guthrie: Metall. Mater. Trans. B, 21B (1992), 765.

21) D. Mazumdar and R. I. L. Guthrie: ISIJ Int., 35 (1995), 1. 